\title{
Comparative Study on 3D Capacitance Imaging Sensors
}

\author{
Hua Yan*, Shizhe Liu and Yanhui Sun \\ School of Information Science and Engineering, Shenyang University of Technology, Shenyang 110870, China \\ ${ }^{*}$ Corresponding author
}

\begin{abstract}
Electrical capacitance tomography (ECT) is a relatively mature non-invasively imaging technique that attempts to map dielectric permittivity of materials. Recently, 3D ECT has gained interest because of its potential to generate volumetric images. In this paper, two kinds of 3D capacitance imaging sensors with 12 electrodes are researched by Finite Element simulation. The effect of rotation of the electrodes on the sensor output, sensitivity distributions and image reconstruction is studied. Based on comparative analysis, better sensor structure are determined.
\end{abstract}

Keywords-electrical capacitance tomography; $3 D$ ECT sensor; $3 D$ sensitivity distribution; $3 D$ reconstruction

\section{INTRODUCTION}

Industrial processes rely heavily on two-phase flow phenomena. Electrical capacitance tomography (ECT) is a relatively mature technique for non-invasively imaging gassolid or gas-liquid chemical engineering processes [1,2]. ECT is based on measuring capacitances of a multi-electrode sensor surrounding a vessel or pipe containing materials of different permittivities. Owing to the distinct advantages such as high safety, easy implementation, low cost, and non-intrusive sensing, ECT is considered as a promising monitoring technique.

ECT has been typically used to obtain 2D maps of the permittivity distribution in a process. However, in recent years, 3D ECT [3,4] has gained interest because of its potential to generate volumetric images. Compared with 2D ECT, 3D ECT is more challenging. The reasons for this are serious difficulties in sensor design, reconstruction algorithm and capacitance measurement. In this paper, two kinds of 3D capacitance imaging sensors with 12 electrodes are researched by Finite Element simulation. The effect of rotation of the electrodes on the sensor performance is studied. Based on comparative analysis, better sensor structure are determined.

\section{INTRODUCTION WORKING PRINCIPLE OF ECT SYSTEM}

In an ECT system, an array of $\mathrm{N}$ electrodes is placed around the region to be imaged. In a complete measurement cycle, a excitation potential is first supplied to electrode 1 and the capacitances between 1 and 2, 1 and $3, \ldots$, and 1 and $\mathrm{N}$ are measured. Next, electrode 2 is excited and the capacitances between 2 and 3,2 and $4, \ldots$, and 2 and $\mathrm{N}$ are measured. This process continues until electrode $\mathrm{N}-1$ is excited, and the capacitance between $\mathrm{N}-1$ and $\mathrm{N}$ are measured. In this way,
$\mathrm{N}(\mathrm{N}-1) / 2$ independent capacitance measurements are produced in a cycle [5].

Assuming free space charge distribution inside the screen, the electrostatic field in the 3D capacitance imaging sensor is governed by Laplace's equation:

$$
\nabla \cdot(\varepsilon(\mathrm{x}, \mathrm{y}, \mathrm{z}) \nabla \phi(\mathrm{x}, \mathrm{y}, \mathrm{z}))=0
$$

Where $\varepsilon(\mathrm{x}, \mathrm{y}, \mathrm{z})$ and $\phi(\mathrm{x}, \mathrm{y}, \mathrm{z})$ are the permittivity distribution and the potential distribution respectively. Under the Dirichlet conditions, i.e. the known potentials of all the electrodes and the screen, the numerical solution of equation (1) can be obtained by using the Finite Elememnt method. From the solution, i.e. nodal potential values, the induced charges on each electrode and subsequently the capacitance values can be calculated. In this paper, the 3D FE models of the ECT sensors are established by using COMSOL software.

\section{3D CAPAXITANCE IMAGING SENSORS}

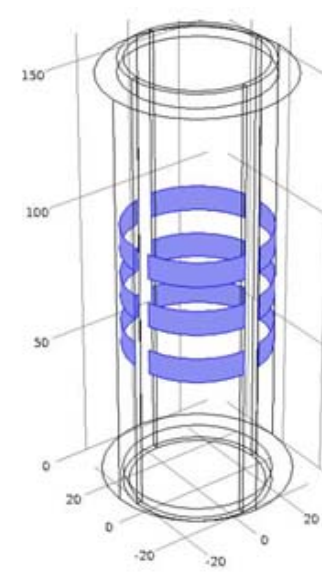

SENSOR 1

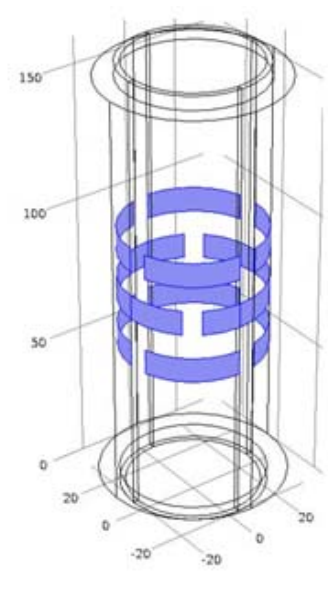

SENSOR 2
FIGURE I. 3D CAPACITANCE IMAGING SENSORS.

The 3D capacitance imaging sensors researched in this paper have twelve electrodes. Fig. I shows the sensor models established in Comsol Multiphysics platform. These twelve electrodes arranged in three planes, each with four electrodes. Every four electrodes on each plane are rotated $0^{\circ}$, we name it sensor 1 or $45^{\circ}$, we name it sensor 2 with reference to the previous, see Fig. I. The length of the pipe is $160 \mathrm{~mm}$, the inner 
and outer diameters of the pipe are $46 \mathrm{~mm}$ and $50 \mathrm{~mm}$, respectively. The pipe wall is assumed to have a relative permittivity of 3.5 . Each electrode is $9 \mathrm{~mm}$ in height and $32.725 \mathrm{~mm}$ in width. The gap length between electrodes in horizontal direction is $6.545 \mathrm{~mm}$ and in vertical direction is $9 \mathrm{~mm}$. The shield screen is $64 \mathrm{~mm}$ in diameter and $150 \mathrm{~mm}$ in length. Fig. II describe the layout of these electrodes in two dimension.

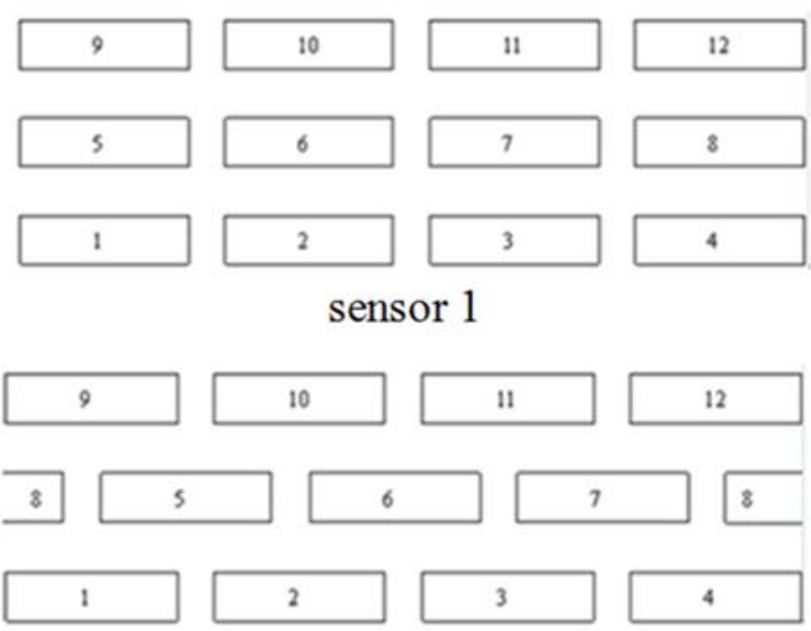

sensor 2

FIGURE II.

ELECTRODE LAYOUTS IN TWO DIMENSION

\section{ReCOnStRuction ALgORITHM}

From the capacitance measurements, images can be reconstructed by using the Linear Back Projection (LBP) algorithm. The LBP algorithm [5] is the most commonly used ECT reconstruction algorithm since it is simple and fast. Using the LBP algorithm, the grey-level of the eth pixel of the reconstruction image can be expressed as:

$$
G(e)=\frac{\sum_{i=1}^{N-1} \sum_{j=i+1}^{N} \frac{C_{i, j}^{m}-C_{i, j}^{l}}{C_{i, j}^{h}-C_{i, j}^{l}} S_{i, j}(e)}{\sum_{i=1}^{N-1} \sum_{j=i+1}^{N} S_{i, j}(e)}
$$

Where $C_{i, j}^{m}$ is the capacitance measurement of electrode pair $i-j, C_{i, j}^{l}$ and $C_{i, j}^{h}$ are the capacitances of electrode pair $i-j$ when the imaging region is filled with material of low permittivity $\left(\varepsilon_{l}\right)$ and high permittivity $\left(\varepsilon_{\mathrm{h}}\right)$, respectively.

The images obtained by the LBP algorithm are edge blur. In order to reduce the fuzzy phenomenon, we use an adaptive thresholding as follows.

$$
\begin{gathered}
G(e)= \begin{cases}0, & G(e)<\eta \\
G(e), & 1>G(e) \geq \eta \\
1, & G(e) \geq 1\end{cases} \\
\eta=\min _{i}\left\|\mathbf{C}-\mathbf{S G}_{i}^{t}\right\|_{2}^{2} \mathbf{G}_{i}^{t}=\left\{\begin{array}{l}
0, \mathbf{G}<\mathrm{i} / \mathrm{L} \\
1, \mathbf{G} \geq \mathrm{i} / \mathrm{L}
\end{array}\right.
\end{gathered}
$$

Where $i \in 1, \ldots, \mathrm{L}$. That means the optimal threshold value is obtained by considering all possible thresholds and choosing the threshold which has the minimum error from the measured values. In this paper, $L=100$. The calculation of the optimal threshold is very fast when $L=100$.

\section{CRITERIA FOR EVALUATING RECONSTRUCTION QUALITY}

In this paper, spatial image error (SIE), relative image error (RE) and correlation coefficient (CC) are used to evaluate the reconstruction quality quantitatively.

$$
\operatorname{SIE}=\frac{\sum_{\mathrm{e}=1}^{\mathrm{M}}\left|\mathrm{G}_{\mathrm{t}}(\mathrm{e})-\mathrm{G}_{\mathrm{r}}(\mathrm{e})\right|}{\sum_{\mathrm{e}=1}^{\mathrm{M}} \mathrm{G}_{\mathrm{t}}(\mathrm{e})}
$$

where Gt(e) and $\operatorname{Gr}(\mathrm{e})$ refer to the true and reconstructed distributions as follows.

$$
\mathbf{G}_{\mathrm{t}}(\mathrm{e})=\left\{\begin{array}{l}
1, \text { if the eth element in the true distribution is } \\
\text { occupied by high permittivity material } \\
0 \text {, otherwise }
\end{array}\right.
$$

$$
\mathbf{G}_{\mathrm{r}}(\mathrm{e})=\left\{\begin{array}{l}
1, \text { if the grey level of the eth element in the } \\
\text { reconstruction is non-zero } \\
0, \text { otherwise }
\end{array}\right.
$$

$$
\mathrm{RE}=\frac{\left\|G-G_{t}\right\|}{\left\|G_{t}\right\|}
$$

$$
C C=\frac{\sum_{\mathrm{e}=1}^{\mathrm{M}}\left(\mathbf{G}_{\mathrm{t}}(\mathrm{e})-\overline{\mathbf{G}}_{\mathrm{t}}\right)(\mathbf{G}(\mathrm{e})-\overline{\mathbf{G}})}{\sqrt{\sum_{\mathrm{e}=1}^{\mathrm{M}}\left(\mathbf{G}_{\mathrm{t}}(\mathrm{e})-\overline{\mathbf{G}}_{\mathrm{t}}\right)^{2}} \sqrt{\sum_{\mathrm{e}=1}^{\mathrm{M}}(\mathbf{G}(\mathrm{e})-\overline{\mathbf{G}})^{2}}}
$$

Where $\mathrm{M}$ is the number of the voxels of the whole imaging region; $\mathbf{G}_{\mathrm{t}}$ and $\mathbf{G}$ are the vectors describing the real and reconstructed permittivity distributions respectively. $\mathbf{G}$ is usually called 'grey vector'; $\overline{\mathbf{G}}$ and $\overline{\mathbf{G}}$ are the mean of $\mathbf{G}_{\mathbf{t}}$ and 
G respectively. Obviously smaller SIE, smaller RE, and CC closer to 1 mean better reconstruction quality.

\section{COMPARISON OF SENSOR PERFORMANCE}

\section{A. Sensor Outputs}

The number of outputs of a capacitance imaging sensor with 12 electrodes is 66 . When the sensor is filled with the air, the capacitance measurements are called empty capacitances $\left(\mathrm{C}_{\mathrm{e}}\right)$. While when the senor is full of high permittivity material ( $\varepsilon_{\mathrm{h}}=2.4$ in this paper), the capacitance measurements are called full capacitances $\left(\mathrm{C}_{\mathrm{f}}\right)$. The comparison of senor outputs when the sensors are empty and full are given in TABLE I and Fig. III. In TABLE I, $\mathrm{C}_{\mathrm{fmax}}$ and $\mathrm{C}_{\mathrm{emin}}$ represent the maximum and the minimum output among the 66 capacitance outputs when the sensor if full of air and high permittivity materials respectively.
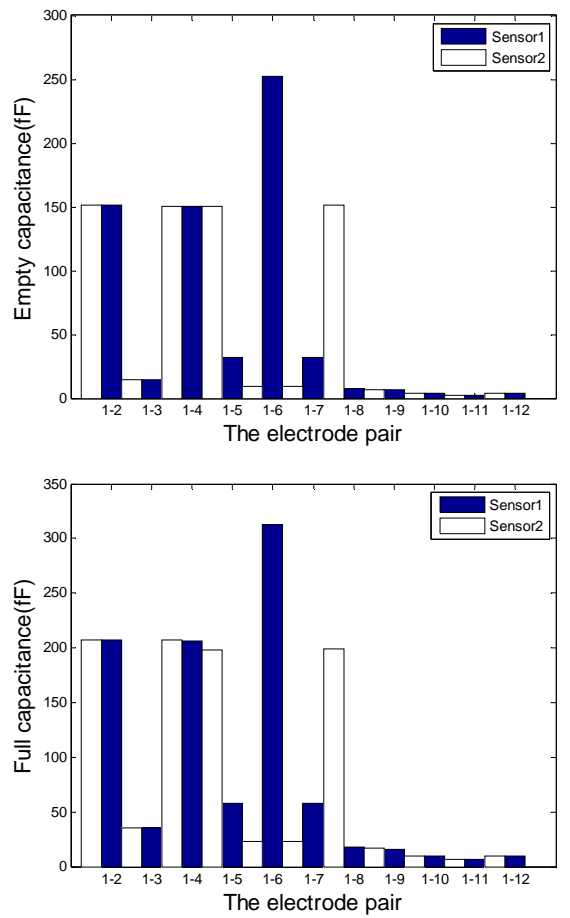

FIGURE III.

COMPARISON OF THE SENSOR OUTPUTS

TABLE I. COMPARISON OF THE SENSOR OUTPUTS

\begin{tabular}{|c|c|c|c|}
\hline & $\mathbf{C}_{\text {fmax }}$ & $\mathbf{C}_{\text {emin }}$ & $\mathbf{C}_{\text {fmin }} / \mathbf{C}_{\text {emin }}$ \\
\hline Sensor 1 & $\begin{array}{c}316.6606 f \mathrm{fF} \\
\text { (electrode pair 5-6) }\end{array}$ & $\begin{array}{c}2.5301 \mathrm{fF} \\
\text { (electrode pair 1-11) }\end{array}$ & 125.156 \\
\hline Sensor 2 & $\begin{array}{c}\text { 207.1582fF } \\
\text { (electrode pair 1-2) }\end{array}$ & $\begin{array}{c}\text { 2.5299fF } \\
\text { (electrode pair 2-12) }\end{array}$ & 81.883 \\
\hline
\end{tabular}

As can be seen from TABLE I and Fig. III, the minimum outputs of sensor 1 and sensor 2 are very close, while the maximum output of sensor 1 is much bigger than that of sensor
2, which means the capacitance measuring for sensor 2 is easier than that for sensor 1 due to its smaller dynamic range.

\section{B. Sensing Fields}

ECT sensors have inhomogeneous three dimensional sensing fields. Usually, the sensitivity distributions of the sensors are used to described the sensing fields of the sensors. Subdividing the space into $M$ voxels, the sensitivity of kth voxel of electrode pair i-j can be defined as:

$$
\mathrm{s}_{\mathrm{ij}}(\mathrm{k}) \cong-\int_{\mathrm{V}_{\mathrm{k}}} \frac{\mathrm{E}_{\mathrm{i}}(\mathrm{x}, \mathrm{y}, \mathrm{z}) \cdot \mathrm{E}_{\mathrm{j}}(\mathrm{x}, \mathrm{yz})}{\mathrm{V}_{\mathrm{C}}^{2}} \mathrm{dxdydz}
$$

where $E_{i}(x, y, z), E_{j}(x, y, z)$ are the electric field strength distribution when the excitation voltage $V_{c}$ is applied to ith electrode and jth electrode, respectively, $\mathrm{V}_{\mathrm{k}}$ is the kth voxel. In this paper, the $46 \mathrm{~mm} \times 46 \mathrm{~mm} \times 55 \mathrm{~mm}$ space enclosing the twelve electrodes is digitized into $32 \times 32 \times 28$ voxels, each voxel is $1.43 \mathrm{~mm} \times 1.434 \mathrm{~mm} \times 2 \mathrm{~mm}$. That means the space is divided into 28 sections, with 1024 voxels of each section. The voxels outside the pipe should be removed, thus the voxels in each section become 762, the total number of voxels to be imaged is 21336. For each sensor, we calculated the sensitivities of these 21336 voxels and constituted a sensitivity matrix with the results. Some comparison of the sensing fields of sensor 1 and 2 are given in Fig. IV.

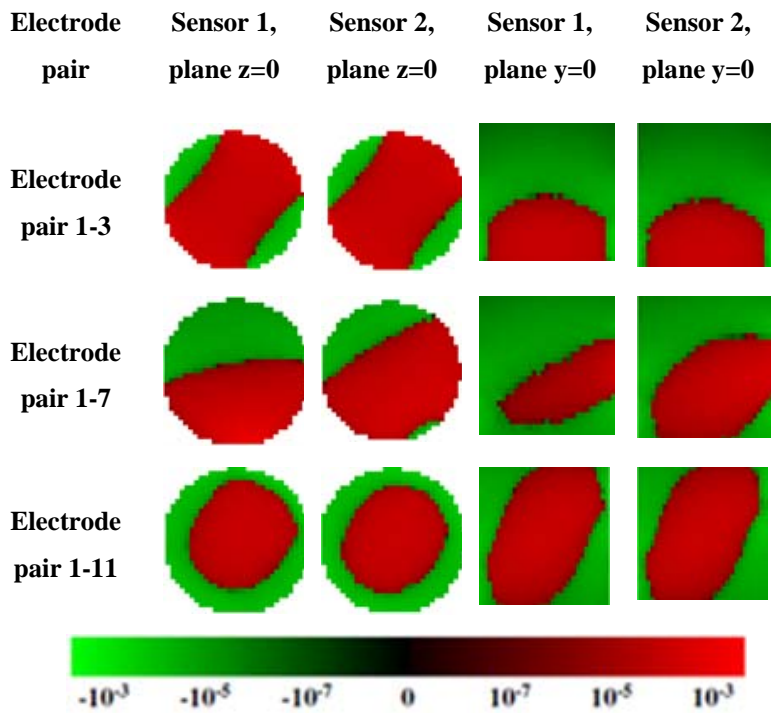

FIGURE IV

COMPARISON OF THE SENSING FIELDS

The condition number and the minimum singular value of the normalized sensitivity matrix is important evaluations of the anti-jamming performance of the sensor. They are judgments of whether the matrix is ill-conditioned. The greater the condition number or the smaller the minimum singular value, the more serious the ill-conditioned. The condition number and the minimum singular value of the normalized sensitivity matrix of sensor 1, 2 are given in TABLE II. As can be seen from TABLE II, sensor 2 corresponds smaller 
condition number and bigger minimum singular value. Therefore, from the view of anti-interference performance, sensor 2 is better.

TABLE II. CONDITION NUMBER AND Minimum SingUlar VAlUE

\begin{tabular}{|c|c|c|}
\hline & Sensor 1 & Sensor 2 \\
\hline Condition number & 113.4433 & 46.9724 \\
\hline Minimum singular value & 0.00067215 & 0.0016 \\
\hline
\end{tabular}

\section{Reconstructed Quality.}

Various Image reconstructions have been conducted by using the LBP algorithm and the capacitance values calculated by the FE models of sensor 1 and sensor 2 . The true objects, the reconstructed objects and the reconstruction errors are given in TABLE III. The objects are given in two ways. One is the $3 \mathrm{D}$ view (contour-surface) and the other is the cross section in plane $y=0$. The capacitances are noise-free FE simulation values. Viewing from the reconstructed images and the reconstruction errors given in TABLE III, the difference between the two sensors are not obvious.

TABLE III. THE TRUE OBJECTS,THERECONSTRUCTED OBJECTS AND THE RECONSTRUCTION ERRORS

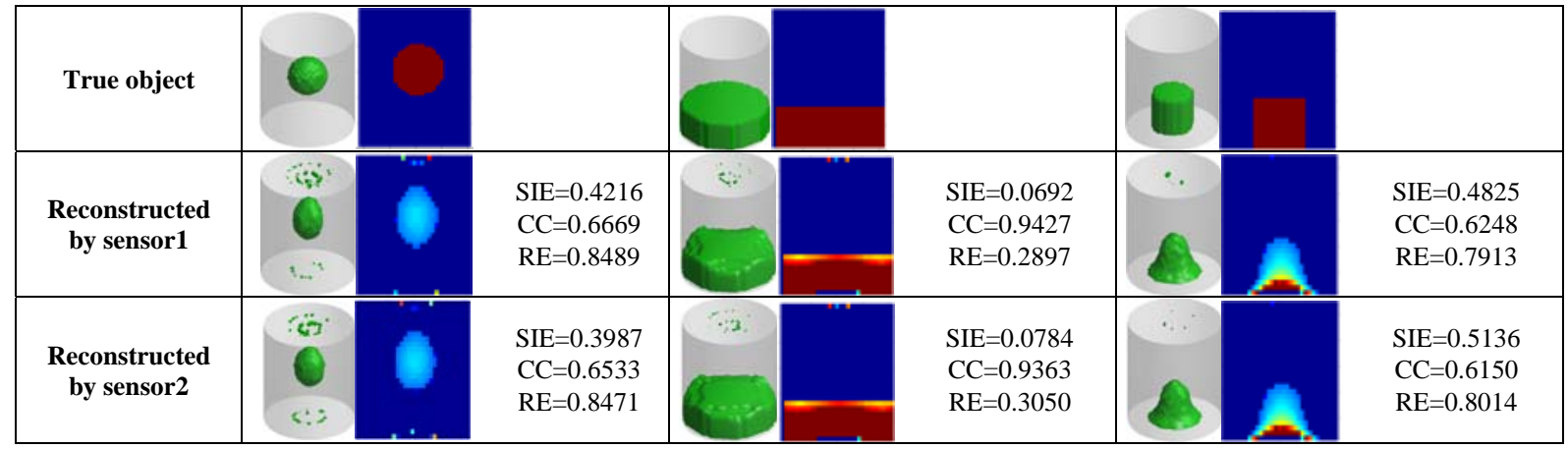

\section{SUMMARY}

The performance of an ECT sensor depends upon its structure and parameters. Two 12-electrode 3D ECT sensors which have the same coverage region of electrodes are investigated and compared. The electrodes are arranged in three plane in two manners. Every four electrodes on each plane are rotated $0^{\circ}$, we name it sensor 1 or $45^{\circ}$, we name it sensor 2 with reference to the previous. Following can be found in the research results based on the Comsol Multiphysics platform.

1) The capacitance measuring for sensor 2 is easier than that for sensor 1 .

2) Sensor 2 corresponds smaller condition number and bigger minimum singular value. Therefore, from the view of anti-interference performance, sensor 2 is better.

3) When the capacitance outputs are noise-free, sensor 1 and sensor 2 give similar reconstruction quality.

Therefore, we can conclude that sensor 2 is better than sensor 1 .

\section{ACKNOWLEDGEMENT}

The authors wish to thank the National Natural Science Foundation of China (No. 61071141), the Program for Liaoning Excellent Talents in University (No.LR2013005) for supporting this research.

\section{REFERENCES}

[1] F. Wang, Q. Marashdeh, L. S. Fan, and W. Warsito, "Electrical capacitance volume tomography: design and applications, ” Sensors, pp. 1890-1917, October 2010

[2] W. Warsito, L. S. Fan, "ECT imaging of three-phase fluidized bed based on three-phase capacitance model, ” Chemical Engineering Science,vol. 58,2003, pp.823-832.

[3] H. Yan, Y. F. Wang, Y. G. Zhou and Y. H. Sun, "3D ECT reconstruction by an improved Landweber iteration algorithm, " Flow Measurement and Instrumentation, vol. 37,2014, pp. 92-98.

[4] R. Wajman, P. Fiderek, H. Fidos, T. Jaworski, J. Nowakowski, D. Sankowski, and R. Banasiak, "Metrological evaluation of a 3D electrical capacitance tomography measurement system for two-phase flow fraction determination,”Meas.Sci. Technolvol.24,2013, doi:10.1088/0957- 0233/24/6/065302.

[5] C. G. Xie, S. M. Huang, B. S. Hoyle, "Electrical capacitance tomography for flow imaging: system model for development of image reconstruction algorithms and design of primary sensors," IEE Proceedings G, vol. 39,1992,pp.89-98. 